\title{
Oncolytic viruses as immunotherapy: progress and remaining challenges
}

This article was published in the following Dove Press journal:

OncoTargets and Therapy

4 May 2016

Number of times this article has been viewed

\section{Laure Aurelian}

Department of Pharmacology, University of Maryland School of Medicine, Baltimore, MD, USA

Correspondence: Laure Aurelian Department of Pharmacology, University of Maryland School of Medicine, 655 W. Baltimore Street, Baltimore, MD 2120I, USA

Email laurelian@som.umaryland.edu
Abstract: Oncolytic viruses (OVs) comprise an emerging cancer therapeutic modality whose activity involves both direct tumor cell lysis and the induction of immunogenic cell death (ICD). Cellular proteins released from the OV-lysed tumor cells, known as damage-associated molecular patterns and tumor-associated antigens, activate dendritic cells and elicit adaptive antitumor immunity. Interaction with the innate immune system and the development of longlasting immune memory also contribute to OV-induced cell death. The degree to which the ICD component contributes to the clinical efficacy of OV therapy is still unclear. Modulation of a range of immune interactions may be beneficial or detrimental in nature and the interactions depend on the specific tumor, the site and extent of the disease, the immunosuppressive tumor microenvironment, the OV platform, the dose, time, and delivery conditions, as well as individual patient responses. To enhance the contribution of ICD, OVs have been engineered to express immunostimulatory genes and strategies have been developed to combine OV therapy with chemo- and immune-based therapeutic regimens. However, these approaches carry the risk that they may also be tolerogenic depending on their levels and the presence of other cytokines, their direct antiviral effects, and the timing and conditions of their expression. The contribution of autophagy to adaptive immunity, the ability of the OVs to kill cancer stem cells, and the patient's baseline immune status are additional considerations. This review focuses on the complex and as yet poorly understood balancing act that dictates the outcome of OV therapy. We summarize current understanding of the OVs' function in eliciting antitumor immunity and its relationship to therapeutic efficacy. Also discussed are the criteria involved in restraining antiviral immune responses and minimizing pathology while promoting antitumor immunity to override immune tolerance.

Keywords: innate and adaptive immunity, autophagy, cancer stem cells, programmed cell death, immunogenic cell death, immunosuppression

\section{Introduction}

The past two decades have witnessed increased interest in oncolytic viruses (OVs) as cancer therapeutic agents. To date, 20 virus platforms have been studied and new candidates continue to emerge for each platform. The first OV to generate positive results in a Phase III clinical trial is derived from the herpes simplex virus (oHSV) and is known as talimogene laherparepvec (T-VEC). ${ }^{1}$ The US Food and Drug Administration (FDA) approved it for the treatment of advanced metastatic melanoma on October 27, 2015, under the name of Imlygic (Amgen Inc, Thousand Oaks, CA, USA). Other OVs completed early (Phase I or I/II) clinical trials for glioblastoma multiforme (a frequent adult brain tumor with dismal prognosis), ${ }^{2}$ other solid tumors (including breast, colon, pancreas, prostate, head and neck, ovaries, lung, and kidney cancer), ${ }^{3}$ as well as leukemia and lymphoma. ${ }^{4}$ Importantly, all the OVs studied so 
far have a near-complete absence of serious adverse events. Unfortunately, however, although the results differed for the various virus platforms, efficacy was limited and often did not exceed that seen for gold standard drug therapy. ${ }^{5}$ This limitation was clearly recognized by the FDA in its recent T-VEC approval.

An important aspect that is often ignored in the effort to overcome this limitation is the recognition that clinical efficacy is a delicate balance among the following: 1) effective OV replication and virus clearance by the induced antiviral immunity; 2) antitumor immunity and factors promoting tumor growth; and 3) immune stimulation and immunosuppressive characteristics of the tumor microenvironment. As altering any one of these parameters may counteract the positive effect of the other parameters, a better understanding of these mechanisms is of utmost importance. Armed with such knowledge, the development of OVs that have multimodal cooperative and coamplifying antitumor activities becomes a feasible future approach. Herein, the current findings in this field and their implications are briefly reviewed. Topics dealt with include cancer stem cell (CSC) lysis and autophagy, modulation of innate and adaptive immunity, immunogenic cell death (ICD), OV transgene arming for improved clinical efficacy, inhibition of the immunosuppressive tumor microenvironment, OV delivery, and combinatorial therapy.

\section{CSCs as targets of OV therapy: autophagy and immunity connection}

A cancer cell subset known as CSCs is highly tumorigenic and has stem cell-like properties, including phenotypic/molecular markers, growth in spheroid culture, and the ability to differentiate into cancer cells. They differ from normal stem cells in that their self-regeneration capacity is deregulated. ${ }^{6} \mathrm{CSC}$ s contribute to drug resistance, metastasis, and tumor recurrence, but the exact mechanism(s) and potential relationship to immune modulation are still poorly understood. Recent findings indicate that glioma cells exposed to the chemotherapeutic agent temozolomide interconvert between nonglioma and glioma stem cells, thereby replenishing the original tumor population and leading to a more infiltrative phenotype, as well as enhanced chemoresistance. ${ }^{7}$ Conventional chemotherapy and radiation may also induce stemness in non-CSCs, ${ }^{8}$ and a multilayered relationship between CSCs and tumorassociated macrophages may be a key component of the immunosuppressive tumor microenvironment. ${ }^{9}$
OVs trigger cancer cell lysis through their preferential replication in tumor as opposed to normal cells and they induce programmed cell death pathways, such as apoptosis, necroptosis, and autophagic cell death, which also contribute to tumor eradication. At least some of these death pathways also have an immunogenic component (Table 1). OVs developed from various virus platforms have been also shown to lyse cells with CSC properties, ${ }^{10-13}$ but the exact contribution of CSC lysis to OV-induced antitumor immunity and its mechanism are still poorly understood. Autophagy plays a crucial role in CSC survival, tumor growth, and drug resistance. ${ }^{14-16}$ It is a major factor in colorectal and breast cancer CSC maintenance and tumor development in athymic mice, ${ }^{17,18}$ potentially involving interleukin (IL)-6, ${ }^{16}$ and promotes the dynamic equilibrium between CSCs and non-CSCs in pancreatic cancer. ${ }^{19}$ Similarly, a CSC subset in urinary bladder cancer has been shown to have autophagy-related increased expression of stemness genes and spheroid growth capacity that appeared to contribute to drug resistance, ${ }^{20}$ suggesting that OV-induced autophagy (potentially through immune modulation) could be clinically detrimental. By contrast, the ability of autophagy to improve antigen cross-presentation of tumor-associated antigens (TAAs) released from OV-lysed tumor cells ${ }^{21}$ could be used to enhance OV clinical efficacy. ${ }^{22,23}$ Significantly, autophagy also plays an important role in maintaining a dynamic equilibrium between CSCs and normal stem cells. ${ }^{19}$ It contributes to the maintenance and function of normal hematopoietic stem cell and affects $\mathrm{T}$-cell polarization, targeting various $\mathrm{T}$-cell subsets, including regulatory T-cells (Tregs), and immune networks at specific anatomical sites. ${ }^{24}$ Indeed, the autophagy response varies according to the cell type, stress, and stimulus and is affected by the tumor microenvironment. While the effect of these factors on CSC modulation is still poorly understood, these currently available data underscore the importance of the CSC in OV-mediated therapy.

Table I Mechanisms of OV-induced cell death and immunogenicity

\begin{tabular}{ll}
\hline Type of cell death & Immunogenicity \\
\hline Necrosis & Releases DAMPs and TAAs; induces ICD \\
Apoptosis & Generally nonimmunogenic \\
Pyroptosis & Caspase-I-dependent cytokine release; \\
& induces ICD \\
Autophagic cell death & Releases DAMPs; immunogenic \\
\hline
\end{tabular}

Notes: OVs induce multiple tumor cell death pathways, most of which are also immunogenic. DAMPs and TAAs released from OV-infected cells induce immunogenic cell death.

Abbreviations: DAMP, damage-associated molecular pattern; ICD, immunogenic cell death; o, oncolytic; OV, oncolytic virus; TAA, tumor-associated antigen. 


\section{Innate immunity contributes to OV therapy}

Viral proteins and nucleic acids are distinguished from cellular counterparts by pattern recognition receptors, such as toll-like receptors (TLRs), located in the cytoplasm or on the cell surface. Their engagement induces expression of inflammatory cytokines (viz, interferon [IFN]- $\alpha,-\beta,-\gamma$, tumor necrosis factor [TNF]- $\alpha$, IL-6, and IL-12), which bind to receptors on other cells, resulting in the production of antiviral genes and immune cell recruitment. Innate immunity is present in all individuals at all times, but is not antigen specific, does not lead to lasting immune memory, and does not increase with repeated exposure to the antigen.

Macrophages, neutrophils, natural killer (NK) cells, and dendritic cells (DCs) are key participants in innate immunity. They can recognize the presence of a stromally remodeled growing tumor, leading to the induction of inflammatory signals and the recruitment of lymphoid cells to the tumor site. A parvovirus OV was shown to activate DCs, partially through TLR 3 and TLR $9,{ }^{25}$ and a reovirus OV escaped the DC endosomes and triggered non-TLR3 receptors to induce IFN- $\gamma$ production, priming for adaptive antitumor immunity. ${ }^{26}$ In a non-small-cell lung carcinoma, intratumoral injection of a coxsackievirus B3 OV caused a major increase in the numbers of NK cells, and the OV therapeutic efficacy was significantly reduced by NK cell depletion. ${ }^{27}$ Intratumorally delivered lipopolysaccharide, a TLR4 agonist, activated innate immune pathways and enhanced the local efficacy of a vesicular stomatitis virus OV (oVSV) genetically modified to express the artificial TAA ovalbumin (OVA), apparently through co-recruitment of antitumor adaptive T-cell responses..$^{28}$ However, this can also sensitize the host to a cytokine shock-like response, particularly if the OV is systemically delivered. ${ }^{28} \mathrm{~A}$ concern is that the existing innate immunity could destroy the OV or limit its spread before it has had the chance to replicate and induce cytotoxicity of a magnitude sufficient to release damage-associated molecular patterns (DAMPs)/TAAs that set up an effective adaptive immunity-based vaccination response. ${ }^{29,30}$ Indeed, chronic activation of innate immunity correlates with poor prognosis, at least in patients treated with an adenovirus OV (oAdV), ${ }^{31}$ and additional studies are needed to better understand the role of innate immunity in OV therapy.

\section{Priming for adaptive immunity plays a critical role in OV therapy}

Induction of an adaptive immune response begins when DCs ingest an antigen and then migrate to the peripheral lymphoid tissues, wherein they mature into antigen-presenting cells that express both the antigen and the costimulatory molecules necessary to activate naïve T-lymphocytes. The antigenactivated lymphocytes give rise to clones of antigen-specific effector cells that mediate adaptive immunity. Unlike innate immunity, the adaptive immune response generates immune memory, meaning that any subsequent exposure to the antigen will induce an immediate, stronger response.

Priming for adaptive immunity plays a critical role in OV antitumor activity, as evidenced by the complete abolishment of the therapeutic effect of a Newcastle disease virus OV (oNDV) in glioma, through depletion of $\mathrm{CD} 8^{+}$ T-cells and the ability of the long-term-surviving mice to resist secondary exposure to glioma, but not other tumor types. ${ }^{32}$ Interaction with innate immunity is suggested by the finding that the parvovirus OV H-1PV increased DC expression of TLR3 and TLR9 in a syngeneic mouse model, resulting in TNF- $\alpha$ production and the apparent stimulation of adaptive immunity to viral antigens. The antiviral memory response is of particular concern because it prevents retreatment, which is an integral component of therapy. ${ }^{33}$ However, OV-induced immune memory to host antigens is a dominant function in OV therapy. This is exemplified by the finding that immunocompetent mice treated with a parvovirus OV did not develop GL261 glioma (associated with DC activation and increased release of TNF- $\alpha$ and IL-6) and the long-term survivors failed to develop tumors when rechallenged with uninfected GL261 cells. However, tumors developed in a similarly treated and rechallenged immunodeficient animal model. ${ }^{34}$ Demonstrating the role of host antigens in the therapeutic efficacy of the OVs, mice bearing OVA-modified B16 melanomas were successfully treated with an OVA-VSV and they developed potent antiOVA immunity. ${ }^{35}$ Moreover, a prime-boost experiment, in which injection of an OVA-modified Semliki Forest virus was followed by injection with an OVA-modified vaccinia virus (oVV) showed that shifting the dominance of the OV-induced immune response away from the virus antigens and toward the host antigens, can increase antitumor activity. ${ }^{36}$ Similarly, injection of mice bearing metastatic B16 melanoma with human dopachrome tautomerase (hDCT)modified VSV elicited a strong T-cell response toward viral antigens, but a prime-boost regimen (prime with AdV-hDCT; boost with VSV-hDCT) completely polarized the adaptive immune response toward the hDCT tumor antigen and cured a large percentage of the mice. ${ }^{37}$

Unfortunately, virus-specific adaptive immunity, result ing from a previous natural infection or earlier $\mathrm{OV}$ 
treatment, could limit tumor cell lysis, thereby interfering with the release of DAMPs-and-TAAs and the generation of antitumor immunity. Restraining antiviral immunity and minimizing pathology while promoting antitumor immunity is a complex and poorly understood balancing act that will ultimately dictate the outcome of OV therapy. An additional concern is the possibility that some OVs may also infect immune cells, thereby rendering them nonfunctional. In tumors with high immunologic activity at baseline, targeting immunosuppression is a major goal, which may differ for various virus platforms. For instance, adaptive antiviral immunity was shown to contribute to the clinical efficacy of oHSV, but it reduced the antitumor responses generated by oVSV. ${ }^{38}$ OVs that can skew the immune profile of the tumor microenvironment from immunosuppressive to inflammatory may be particularly effective, as shown for oHSVs armed with IL-12/angiostatin, which limit CSC-mediated neovascularization and recruitment of tumor-supportive tumor-associated macrophages. ${ }^{13}$

\section{OVs as antitumor vaccines: the ICD connection}

OVs induce tumor cell death through a variety of mechanisms, virtually all of which can be actively immunostimulatory (Table 1). This appears to involve release of DAMPs and TAAs from the lysed tumor cells and their cross-presentation into the immune system, leading to the activation of antitumor immunity (Figure 1). ${ }^{39,40}$ Major DAMPs include high-mobility group box protein-1 (HMGB1) (which has proinflammatory properties), heat shock proteins (viz, Hsp70), and uric acid (which is also increased by stressinduced nucleic acid degradation). OVs developed from various virus platforms have this antigen cross-presentationrelated ability to induce antitumor immunity, ${ }^{27,41-44}$ a cell death process known as ICD. As summarized in Table 2 for the major virus platforms, ICD-related immunostimulatory determinants include DC activation and increased levels of inflammatory cytokines, as well as NK and CD8 ${ }^{+} \mathrm{T}$-cell responses. ICD holds the promise that OVs might function

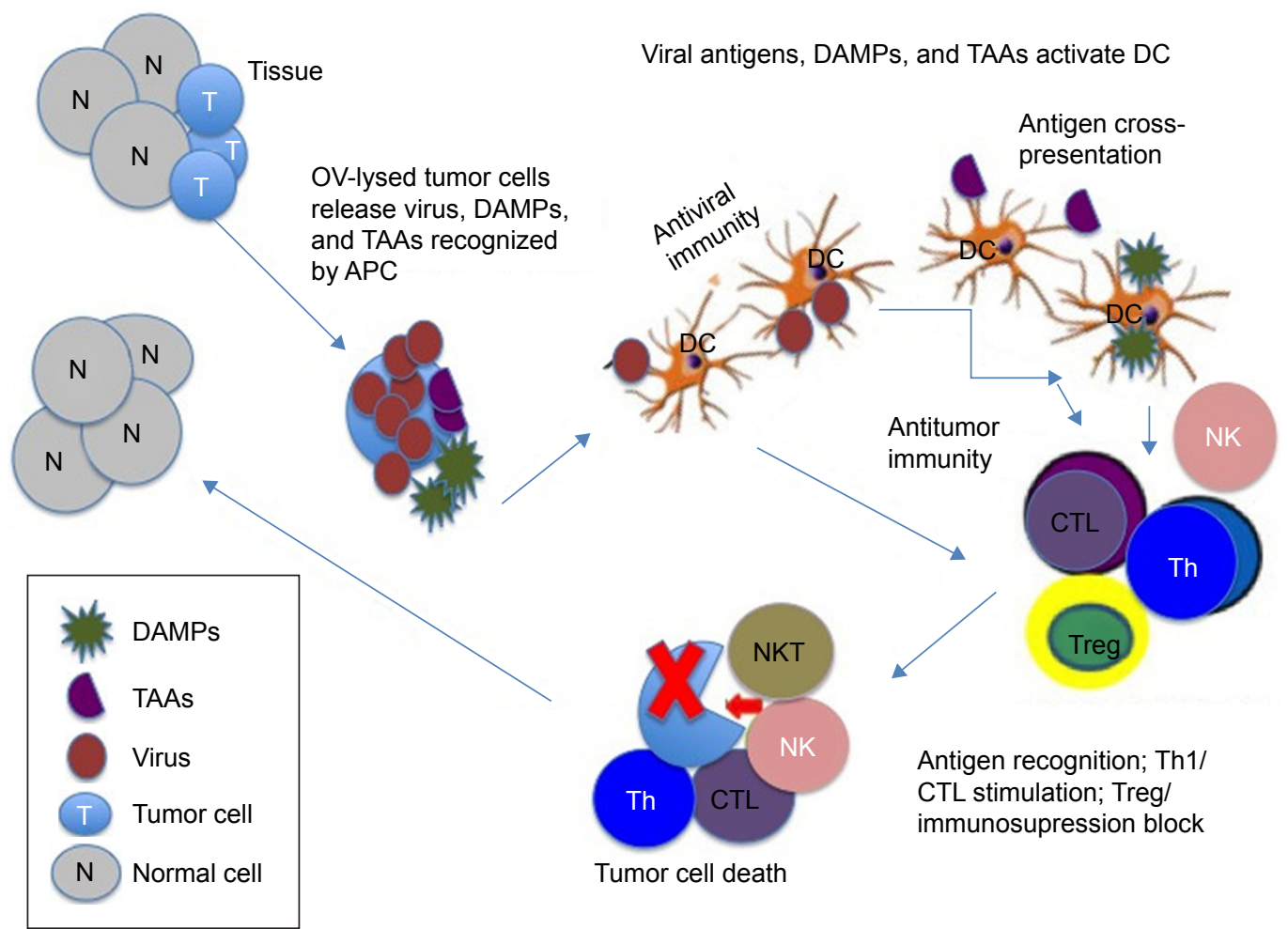

Figure I Schematic representation of OV-induced antitumor immunity.

Notes: OVs are designed to preferentially replicate within tumor cells (T) in the tissue, sparing normal cells (N). This results in tumor cell lysis, release of DAMPs and TAAs, and the activation of immunostimulatory functions that contribute to the development of antitumor immunity. These cellular antigens are recognized by APCs, notably DCs, which are activated in response to the viral antigens (antiviral immunity) and function in antigen cross-presentation. The ensuing immune response includes the activation of T- and NK cells, generation of CTLs, alteration of the Thl/Th2 balance (which inhibits the immunosuppressive tumor microenvironment), and often depletion of Tregs. The resulting stimulatory immune response (ICD) inhibits immunosuppressive conditions and contributes to the eradication of the tumor cells. However, distinct OV platforms differ in their replicative and PCD induction potentials, and they have different abilities to activate these immunostimulatory activities; the modulatory pathways are distinct, particularly in different tumor cells. Accordingly, the development of OVs that function at all these levels is particularly desirable.

Abbreviations: APC, antigen-presenting cell; CTL, cytotoxic T-cell; DAMP, damage-associated molecular pattern; DC, dendritic cell; ICD, immunogenic cell death; NK, natural killer cells; OV, oncolytic virus; PCD, programmed cell death; TAA, tumor-associated antigen; ThI/2, T-helper cell type I or 2; Treg, regulatory T-cell; NKT, natural killer T cells. 
Table 2 OVs induce ICD-like features

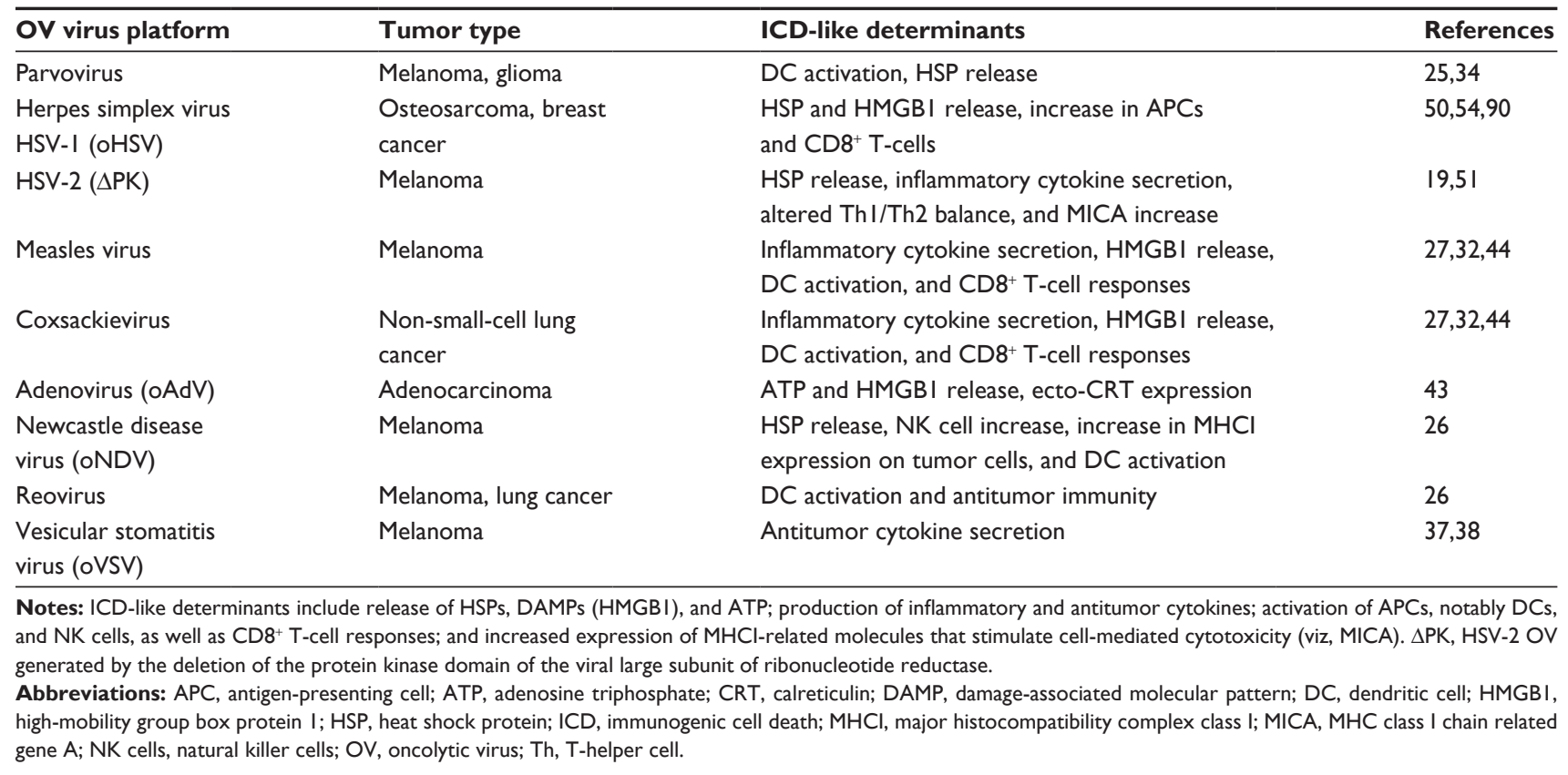

as cancer vaccines..$^{30,45,46}$ Indeed, intratumoral injection of a vaccinia virus $\mathrm{OV}$ (oVV) in an immunocompetent mouse model imparted the capacity to reject rechallenge with the same (but not other) tumor cells, ${ }^{47}$ suggesting that the OVinduced adaptive immunity cross-protected against TAAs (refer section titled "Priming for adaptive immunity plays a critical role in OV therapy").

The degree to which the ICD component contributes to the clinical efficacy of OV therapy is still unclear, but it is reasonable to assume that it depends on the levels of OVinduced tumor cell lysis and differs in distinct tumor types. The site and extent of disease, as well as the specific virus platform, are also likely to affect the relative degree of ICD contribution. ${ }^{48}$ Furthermore, DAMPs and TAAs are not always immunostimulatory. The major DAMP, HMGB1, promote the development of myeloid suppressor cells (MDSCs) and contributes to their ability to suppress T-cell activation. ${ }^{49}$ For instance, in oAdV-treated cancer patients, antitumor immunity was associated with improved clinical outcome when the HMGB1 pretreatment levels were low. ${ }^{50}$ Moreover, DNA damage caused by a defective repair machinery may increase the expression of stress ligands targeted by NK or cytotoxic T-cells, contributing to the effectiveness of OV therapy, independent of ICD. We have recently shown that melanoma tumors treated with the HSV-2 OV known as $\triangle \mathrm{PK}$ express MHC class I chain related gene A (MICA), the ligand for the NKG2D receptor on NK and cytotoxic T-cells, thereby presumably contributing to cytotoxic tumor cell lysis. MICA induction resulted from virus replication and $\mathrm{JNK} / \mathrm{c}$-Jun-dependent inhibition of secretion of the immunosuppressive cytokine IL-10 by melanoma cells. ${ }^{51}$ Further studies are needed to better understand the relative contribution of different death pathways, including ICD, to the OV clinical efficacy.

\section{Transgene arming enhances the OV antitumor activity}

An extensive panel of transgenes has been used to modify the $\mathrm{OV}$ backbone to enhance their therapeutic activity in preclinical studies. This includes inflammatory cytokines, antiangiogenic and antivascular proteins, monoclonal antibodies, proapoptotic genes, and enzymes that degrade extracellular matrix. Clinical data are available for oHSV, oAdV, and oVV, all of them armed with human granulocytemacrophage colony-stimulating factor (GM-CSF), an inflammatory cytokine that bolsters antitumor immune responses by recruiting NK cells and inducing TAA-specific cytotoxic T-cells. ${ }^{45,52}$ The oHSV T-VEC, which was recently approved by the FDA for the treatment of metastatic melanoma, was developed from HSV-1 through deletion of the genes ICP34.5 and ICP47 that are, respectively, involved in virus replication and immune evasion. In Phase I trials, intratumorally delivered T-VEC was generally well tolerated and GM-CSF expression and HSV antigen-associated tumor necrosis were observed. In a Phase II trial, patients' unresectable metastatic melanomas were treated with multiple intratumoral injections 
of T-VEC and monitored for survival and safety. The overall response rate (ORR) based on the Response Evaluation Criteria In Solid Tumors (RECIST) was $26 \%$, with complete response in eight out of 50 patients. ${ }^{53}$ Local and systemic antigen-specific T-cells and decreased immunosuppressive functions, including Tregs and MDSCs, were seen in patients with a therapeutic response. ${ }^{54}$ A pivotal recently completed Phase III study of patients with unresectable stage IIIB-IV melanoma compared intratumoral T-VEC injection to subcutaneous GM-CSF administration. ${ }^{1}$ There were 295 patients in the T-VEC group and 141 in the GM-CSF arm. The overall durable response rate was $16.3 \%$ for T-VEC but only $2.1 \%$ for GM-CSF alone. The objective ORR was $26.4 \%$ for T-VEC and included $10.8 \%$ with a complete response, compared with an ORR of $5.7 \%$ and a complete response of $0.7 \%$ in the GM-CSF group. ${ }^{1}$

Pexa-Vec (also known as JX-594) is an oVV constructed through the deletion of the genes encoding thymidine kinase and vaccinia growth factor. It has undergone multiple Phase I/II clinical trials and clinical responses were seen in liver cancer patients, with median survival of 14.1 and 6.7 months for high and low doses, respectively. ${ }^{55}$ PexaVec induced antibody-mediated complement-dependent cancer cell lysis, which was apparently related to its ability to upregulate the expression of multiple TAAs that were identified by a serological expression cloning assay. ${ }^{56} \mathrm{~A}$ Phase III trial for advanced stage liver cancer was scheduled to begin in late 2015 (http://www.pexavectrials.com). One of the GM-CSF-armed oAdVs that has entered clinical trials, Ad5-D24-GMCSF, induced antitumor immunity in cancer patients, with complete response seen in two and stable disease in five of 16 evaluable patients, ${ }^{57}$ similar results were also obtained for another oAdV..$^{58}$

These studies underscore the effectiveness of the OV-based intervention but fail to contribute to our understanding of the importance of arming the OVs with GMCSF. OVs armed with other cytokines (viz, IL-2, IL-12, IL-15, IL-18, and IFN- $\alpha / \beta$ ), chemokines (viz, CCL5), or costimulatory molecules (viz, B7.1 and CD40L) can also induce antitumor immunity. ${ }^{59}$ For instance, an oHSV armed with IL-12, a potent antitumor cytokine with antiangiogenic activities, reduced neovasculature and Tregs, as well as induced T-cell-mediated immunity in an immunocompetent CSC model. ${ }^{60}$ The chemokine CXCL12 and its receptor CXCR4 are involved in the progression of ovarian cancer through enhancement of tumor angiogenesis and immunosuppressive networks that also regulate CSC development; an oVV armed with a CXCR4 antagonist reduced tumor vasculature and accumulation of suppressor cells, in addition to induction of antitumor humoral responses. ${ }^{61}$ A challenge is that the immunoregulatory factors used to arm the OVs may also contribute to immune tolerance, and the most effective arming approach, particularly as it relates to the OV platform, is unknown. GM-CSF, for instance, has both proinflammatory and tolerogenic functions, depending on the dose and the presence of other cytokines, ${ }^{62}$ and CD40L is effective when incorporated into an oAdV but not into an oVSV. ${ }^{63}$ Moreover, the cytokine-enhanced immunity may inhibit virus replication and, thereby, tumor cell lysis. Strategies to mitigate these concerns include either arming the OVs with chemokines (rather than cytokines), because they influence the overall immune response without having direct antiviral effects, or using weak late promoters to initiate low levels of transgene expression after virus replication has begun. Incorporating exogenous regulators of transgene expression or mRNA/protein stability is another possible approach that induces OV clearance only after an initial period of unhindered oncolytic activity. Once an immune response that limits oncolytic activity is initiated, a second immunotherapeutic phase of viral activity could be enhanced through the use of combinatorial therapy (as discussed in the section "Combinatorial therapy using OVs and pharmacologic or immunotherapeutic regimens"). However, OVs that naturally induce the secretion of multiple inflammatory cytokines (without genetic modification) (Table 3 ) are desirable, particularly if they simultaneously inhibit tumor-promoting immunosuppressive functions, as we have recently shown for the HSV-2 OV, $\triangle$ PK. ${ }^{51}$ Additional studies are needed in order to better understand these contributions, particularly within the context of different tumor types.

\section{The tumor immunosuppressive microenvironment interferes with OV clinical efficacy}

Tumors are composed of cancer and stromal cells and contain infiltrating Tregs and MDSCs, which maintain an immunosuppressive environment and promote tumor growth. ${ }^{64}$ In addition, the tumor cells themselves can secrete immunosuppressive cytokines, such as IL-10, which are involved in the recruitment and activation of MDSCs and the establishment of the premetastatic niche. $.^{65} \mathrm{IL}-10$ also decreases the expression of MICA, the ligand for the activating receptor natural killer group 2 member D (NKG2D) that is expressed on NK, $\gamma \delta \mathrm{T}-$, cytotoxic T-, and NKT cells, thereby reducing cytotoxicity and facilitating tumor cell escape from immune surveillance. ${ }^{66} \mathrm{In}$ hypoxia, which is caused by rapid tumor progression, hypoxia 
Table 3 Strategies to improve OVT clinical efficacy

\begin{tabular}{|c|c|c|}
\hline Virus backbone alterations & Combinatorial therapy & Multimodal activity \\
\hline Deletion of virulence genes & Immune-activating therapy & Kills tumor cells through all death pathways \\
\hline Deletion of immunomodulatory genes & Adoptive T-cell therapy (viz, TCR, CAR T-cell) & (virus replication, $\mathrm{PCD}$, autophagic cell death) \\
\hline Arming with inflammatory or & Immunosuppression inhibition & Induces proinflammatory responses \\
\hline immunosupression-blocking genes or & Blockade of immune checkpoint inhibitors & Inhibits immunosuppressive tumor \\
\hline \multirow[t]{3}{*}{ antiangiogenic or proapoptotic genes } & Chemotherapeutic drugs to delete & microenvironment and induces activators \\
\hline & Tregs/MDSCs or inhibit innate immunity & of NK/T-cell cytotoxicity \\
\hline & & Blocks immune checkpoint regulators \\
\hline
\end{tabular}

Notes: Clinical efficacy can be increased by virus backbone alterations (first column), combinatorial therapy designed to activate the immune response and/or block the immunosuppressive tumor microenvironment (second column), or by the development of OVs with multimodal activity (last column). Instead of using strategies that alter the virus backbone, some studies have used drugs or distinct immunomodulatory methods in combinatorial therapy. OVs with multimodal activities that include cell lysis through virus replication, the induction of multiple PCD pathways, and immunomodulation are particularly desirable.

Abbreviations: CAR, chimeric antigen receptors; MDSC, myeloid suppressor cell; NK, natural killer; OV, oncolytic virus; OVT, oncolytic virotherapy; PCD, programmed cell death; TCR, T-cell receptor; Treg, regulatory T-cell.

inducible factor 1 alpha $($ Hif- $1 \alpha)$ promotes differentiation of Tregs, ${ }^{67}$ and its expression in tumor-infiltrating myeloid DCs upregulates the immune checkpoint regulator programmed death-ligand 1 (PD-L1), further contributing to the immunosuppressive nature of the tumor microenvironment. ${ }^{68}$ Inhibition of this immunosuppressive condition is a major consideration in OV therapy. However, the relevant mechanism that must be targeted is likely to vary in distinct tumor types and could change throughout disease progression. Therefore, targeting only one of the immunosuppressive mechanisms that define the tumor microenvironment is unlikely to be therapeutically effective, unless it can be identified as being dominant in the specific cancer being treated. ${ }^{69}$ This problem is exemplified by the finding that an IL-12-armed oHSV increased survival in a murine glioblastoma stem cell model through reduction of Tregs, but the reduction of Tregs had a negative effect on oVSV treatment, apparently due to the rapid OV clearance that results when the suppression of antiviral immunity was relieved. Depletion of Tregs was also shown to abrogate effective antitumoral T-cell induction by an OV-assisted DC vaccine, ${ }^{22}$ raising new questions about the consequences of Treg depletion on OV-induced antitumoral responses. Because immunosuppressive cytokines that are directly secreted by the tumor cells favor innate immune cells' differentiation into immunosuppressive cells, various combinatorial therapy strategies have been attempted to improve the OV efficacy. However, these carry their own potential risks (refer section on "Combinatorial therapy using OVs and pharmacologic or immunotherapeutic regimens"), and the development of OV platforms that directly inhibit such secretion ${ }^{51}$ is highly desirable (Table 3).

\section{OV delivery affects clinical efficacy}

In addition to the virus platform used in OV development and the specific tumor that is being treated, the route of $\mathrm{OV}$ administration is an important determinant of the initial host response. Intravenous administration is particularly desirable for treatment of metastatic disease. Unfortunately, the $\mathrm{OV}$ is rapidly recognized and eliminated by the circulating antibody and complement molecules, and nonspecific organ/vasculature accumulation and scavenging immune cells curtail tumor targeting and distribution. ${ }^{30}$ Immunemediated OV destruction is probably less effective on intratumoral OV injection, but specific uptake, nontarget binding, and the immunosuppressive tumor microenvironment can still limit OV replication and antitumor activity (refer "The tumor immunosuppressive microenvironment interferes with OV clinical efficacy" section).$^{70}$

Tumor-tropic cell carriers that can also cross the bloodbrain barrier have recently been considered a potential method to direct the OVs to the tumor site..$^{71}$ For instance, neural stem cells loaded with oAdV can target gliomas and reduce tumor growth, but concerns include virus toxicity for the carrier cells, stem cells' tumorigenic potential, and immune rejection of heterologous stem cells. ${ }^{72}$ Various immune cell types were also evaluated for their ability to deliver OVs upon systemic injection. For example, therapeutic efficacy was greatly increased in a mouse model of breast cancer by utilizing ex vivo expanded NKT cells (known as CIK) to deliver an oVV, ${ }^{73}$ and DCs were shown to be effective cell carriers for oncolytic reovirus ${ }^{74}$ and measles virus. ${ }^{75}$ A reovirus OV that was ineffective in a host previously exposed to reovirus enhanced the survival of melanoma-bearing mice and induced robust antitumor and antiviral immunity when delivered in DC carriers. ${ }^{76}$ However, not all interactions between OVs and DCs are synergistic, and an oVSV was shown to interfere with DC functions and abrogate TAA presentation. ${ }^{77}$

Recent efforts have also used mesenchymal stem cells (MSCs) to selectively deliver OVs to the tumor while 
shielding them from neutralizing antibodies and protecting them from sequestration. MSCs were used to deliver a measles virus $\mathrm{OV}$ to hepatocellular carcinoma by tail vein injection of seropositive animals, and MSCs expanded under hypoxic conditions were shown to be good carriers for the delivery of conditionally replicative adenoviruses to tumor cells in the presence of virus-neutralizing antibody. The systemically delivered cell carriers tended to become arrested in the lungs immediately upon infusion, but the arrest was transient, thereby failing to significantly diminish efficacy. ${ }^{78}$ Moreover, MSC viability declined over time in both immunocompetent and immunodeficient tumor-bearing mice, relieving concerns about potential differentiation to suppressor function, independent of the immune status of the host.

A concern, however, is the MSC source, as the significant variation in the secretome and immunomodulatory potency of MSCs from different donors is likely to lead to highly variable outcomes. The duration of MSC persistence required for a sustainable $\mathrm{OV}$ therapeutic effect is also potentially problematic. MSC expression of immunogenic and immunosuppressive factors must be determined at baseline and after stimulation with molecules that mimic the physiological conditions to which the MSC will be exposed in the patient. Biomarkers that predict whether or not a patient is likely to respond to the use of MSC as a therapy in its own right, including sensors that image the MSC secretome, should be explored before and after infection with the OV. Adipose-derived stem cells (ADSCs) have also been investigated as potential OV carriers. ADSCs are permissive for myxoma virus replication, and in vitro coculture of human glioblastoma cells with myxoma virus-infected ADSCs showed cross-infection and concomitant cell death exclusively in the tumor cells. In a xenograft model of glioblastoma, intracranial injection with myxoma virus-infected ADSCs led to successful delivery of the OV to the tumor and a significant increase in survival. ${ }^{46}$

\section{Combinatorial therapy using OVs and pharmacologic or immunotherapeutic regimens}

Circumvention of intracellular and microenvironmental antiviral responses using combinatorial therapy regimens may improve clinical efficacy. While much additional information is needed, a number of such approaches were examined. Briefly, one such approach used histone deacetylase inhibitors (HDACi) to inhibit innate antiviral immune responses (viz, IFN type I) to increase the OV spread and antitumor effects. ${ }^{79}$ When administered before an oHSV, the HDACi valproic acid (VPA) inhibited the innate antiviral
NK response and improved the OV clinical efficacy in an orthotopic glioblastoma mouse model. ${ }^{80}$ Another study ${ }^{81}$ concluded that HDACi and DNA methylation inhibitors enhance OV therapeutic potential by increasing tumor cell immunogenicity through upregulation of TAAs and antigen presentation.

In another approach, the OVs were used together with chemotherapeutic agents. Cyclophosphamide (CPM), paclitaxel, and temozolomide successfully reduced Treg activity when delivered as metronomic (viz, low and repetitive) doses that minimize toxicity and avoid global immunosuppression resulting from administering a single, high dose. At a low dose, CPM had no effect on antiviral immunity but it caused depletion of Tregs, improving the clinical efficacy of an oAdV in glioblastoma ${ }^{82} \mathrm{CPM}$ also showed dose-dependent decrease in brain influx and/or function of NK cells, macrophages, and DCs in a U251 glioma xenotransplant model. ${ }^{83} \mathrm{CPM}$ preconditioning to deplete Tregs also enhanced the therapeutic benefits of reovirus and VSV OVs ${ }^{84}$ and early clinical evaluation of metronomic CPM-and-oAdV combination demonstrated improved antitumor efficacy, resulting from increased cytotoxic T-cells and induced T-helper cell type 1 (Th1)-type immunity. ${ }^{82}$ Metronomic CPM was also shown to deplete Tregs and restore $\mathrm{T}$ - and NK-cell effector function in advanced cancer patients. ${ }^{85}$ However, Tregs may actually be needed for optimal virotherapy results, due to their ability to prevent early OV clearance and/or interfere with MDSC induction in vigorously inflamed tumors, as shown by CPM-promoted antiviral immunity. ${ }^{45}$ Conversely, high-dose CPM may not only enhance virus-mediated cell lysis through widespread immunosuppression of the innate and adaptive antiviral immune responses but also completely abrogate the antitumor immune response.

In preclinical studies, ${ }^{86}$ the combination of oHSV with various MDSC-depleting drugs (viz, gemcitabine, sunitinib, 5-fluorouracil (FU), docetaxel, or retinoic acid) improved survival, being associated with increased antitumoral immunity in the case of gemcitabine. However, successful combinatorial therapy is context dependent, and additional studies are needed to define the optimal therapeutic conditions. For instance, metronomic gemcitabine or 5-FU, together with oAdV, increased virus uptake, and sunitinib enhanced intratumoral replication of an oVSV. In contrast, however, concurrent therapy with 5-FU and HSV-1 inhibited virus replication. ${ }^{87} \mathrm{OV}$ combination with a potent agonist antibody specific for the costimulatory molecule 4-1BB showed improved therapeutic outcomes, ${ }^{88}$ and promising results were obtained when the OVs were combined with an immunomodulatory 
monoclonal antibody that blocks T-cell checkpoint blockade receptors, such as cytotoxic T-lymphocyte-associated protein 4 (CTLA-4) or PD-L1. Combining a tumor-targeted $\mathrm{DC}$ vaccine with ongoing $\mathrm{OV}$-induced tumor inflammation was shown to stimulate potent antitumoral $\mathrm{CD}^{+} \mathrm{T}$-cell responses and marked tumor regression in a model of lung cancer metastasis. ${ }^{89}$ Remaining challenges stem from discernable conflicts between virus- and drug-enabled therapeutic strategies. For instance, viruses require actively dividing cells to maximize their replicative efficiency, while many anticancer agents are cytotoxic or cytostatic with death-inducing or antiproliferative effects, respectively. Modulation of the host immune response through chemotherapy may conflict with the $\mathrm{OV}$ therapeutic function, and further research is needed to better understand the response of various tumors. As stated earlier, the development of OVs with multimodal activities that include the appropriate drug-mediated modulation (Table 3 ) is particularly desirable.

\section{Concluding remarks}

OV therapy holds the promise that it may function as a cancer vaccine strategy. This is based on available data that indicate that DAMPs and TAAs released from the tumor cells lysed by the replicating OVs induce tumor-rejecting anticancer immunity. The FDA approval of the OV T-VEC (Imlygic, Amgen) for the local treatment of unresectable metastatic melanoma is the first clinical and regulatory validation of an $\mathrm{OV}$ as a therapeutic. Approval was based on therapeutic benefit, but Imlygic was not shown to improve overall survival or have an effect on visceral metastases, and the extent to which the immunotherapeutic component actually contributes to therapy is unclear. Indeed, the immune system is a doubleedged sword in the context of virotherapy. Specifically, while the antiviral immune response must be minimized for successful virus growth and retreatment, the anti-TAA response must be stimulated. The latter requires both the modulation of innate and adaptive immunity modes and the alteration of the immunosuppressive tumor microenvironment. A range of $\mathrm{OV}$ modifications and immune interactions may be beneficial or detrimental and they depend on the specific tumor, OV platform, dose, time and delivery conditions, as well as individual patient responses. Immunostimulatory cytokines carry the risk of being tolerogenic depending on their levels and the presence of other cytokines, their direct antiviral effects, as well as the timing and conditions of their expression. DAMPs may actually suppress T-cell activation, as shown for HMGB1, and Tregs may be needed for optimal therapeutic results due to prevention of early OV clearance or the compensatory induction of MDSCs. Naturally occurring tumor-lysing antibodies ${ }^{91}$ are potentially promising innate immunity stimulators that may also contribute to the $\mathrm{OV}$ anti-tumor potential. The contribution of autophagy in adaptive immunity, the ability of the OVs to kill CSCs, and the patient's baseline immune status and prior antiviral immunity are additional considerations that may affect efficacy. Further investigation should focus on how to better restrain antiviral immune responses and minimize pathology while promoting antitumor immunity.

\section{Disclosure}

The author reports no conflicts of interest in this work.

\section{References}

1. Andtbacka RH, Kaufman HL, Collichio F, et al. Talimogene laherparepvec improves durable response rate in patients with advanced melanoma. J Clin Oncol. 2015;33(25):2780-2788.

2. Wollmann G, Ozduman K, van den Pol AN. Oncolytic virus therapy for glioblastoma multiforme: concepts and candidates. Cancer J. 2012; 18(1):69-81.

3. Turnbull S, West EJ, Scott KJ, Appleton E, Melcher A, Ralph C. Evidence for oncolytic virotherapy: where have we got to and where are we going? Viruses. 2015;7(12):6291-6312.

4. Betancourt D, Ramos JC, Barber GN. Retargeting oncolytic vesicular stomatitis virus to human T-cell lymphotropic virus type 1-associated adult T-cell leukemia. $J$ Virol. 2015;89:11786-11800.

5. Weller M, Cloughesy T, Perry JR, Wick W. Standards of care for treatment of recurrent glioblastoma - are we there yet? Neuro Oncol. 2013; 15(1):4-27.

6. Kreso A, Dick JE. Evolution of the cancer stem cell model. Cell Stem Cell. 2014;14:275-291.

7. Auffinger B, Tobias AL, Han Y, et al. Conversion of differentiated cancer cells into cancer stem-like cells in a glioblastoma model after primary chemotherapy. Cell Death Differ. 2014;21:1119-1131.

8. Ghisolfi L, Keates AC, Hu X, Lee DK, Li CJ. Ionizing radiation induces stemness in cancer cells. PLoS One. 2012;7(8):ea43628.

9. Raggi C, Mousa HS, Correnti M, Sica A, Invernizzi P. Cancer stem cells and tumor-associated macrophages: a roadmap for multitargeting strategies. Oncogene. 2016;35(6):671-682.

10. Marcato P, Dean CA, Giacomantonio CA, Lee PW. Oncolytic reovirus effectively targets breast cancer stem cells. Mol Ther. 2009;17(6) 972-979.

11. Mantwill K, Naumann U, Seznec J, et al. YB-1 dependent oncolytic adenovirus efficiently inhibits tumor growth of glioma cancer stem like cells. J Transl Med. 2013;11:216.

12. Colunga A, Bollino D, Schech A, Aurelian L. Calpain-dependent clearance of the autophagy protein $\mathrm{p} 62 / \mathrm{SQSTM} 1$ is a contributor to DeltaPK oncolytic activity in melanoma. Gene Ther. 2014;21(4): 371-378.

13. Smith TT, Roth JC, Friedman GK, Gillespie GY. Oncolytic viral therapy: targeting cancer stem cells. Oncolytic Virother. 2014;2014(3):21-33.

14. Bellodi C, Lidonnici MR, Hamilton A, et al. Targeting autophagy potentiates tyrosine kinase inhibitor-induced cell death in Philadelphia chromosome-positive cells, including primary CML stem cells. J Clin Invest. 2009;119:1109-1123.

15. Jiang H, Gomez-Manzano C, Aoki H, et al. Examination of the therapeutic potential of delta-24-RGD in brain tumor stem cells: role of autophagic cell death. J Natl Cancer Inst. 2007;99:1410-1414.

16. Guan JL, Simon AK, Prescott M, et al. Autophagy in stem cells. Autophagy. 2013;9(6):830-849. 
17. Kantara C, O’Connell M, Sarkar S, Moya S, Ullrich R, Singh P. Curcumin promotes autophagic survival of a subset of colon cancer stem cells, which are ablated by DCLK1-siRNA. Cancer Res. 2014;74:2487-2498.

18. Gong C, Bauvy C, Tonelli G, et al. Beclin 1 and autophagy are required for the tumorigenicity of breast cancer stem-like/progenitor cells. Oncogene. 2013;32:2261-2272.

19. Zhu H, Wang D, Liu Y, et al. Role of the hypoxia-inducible factor-1 alpha induced autophagy in the conversion of non-stem pancreatic cancer cells into CD133+ pancreatic cancer stem-like cells. Cancer Cell Int. 2013;13:119.

20. Ojha R, Jha V, Singh SK, Bhattacharyya S. Autophagy inhibition suppresses the tumorigenic potential of cancer stem cell enriched side population in bladder cancer. Biochim Biophys Acta. 2014;1842: 2073-2086.

21. Meng S, Xu J, Wu Y, Ding C. Targeting autophagy to enhance oncolytic virus-based cancer therapy. Expert Opin Biol Ther. 2013;13(6): 863-873.

22. Uhl M, Kepp O, Jusforgues-Saklani H, Vicencio JM, Kroemer G, Albert ML. Autophagy within the antigen donor cell facilitates efficient antigen cross-priming of virus-specific CD8+ T cells. Cell Death Differ. 2009;16(7):991-1005.

23. Duffy A, Le J, Sausville E, Emadi A. Autophagy modulation: a target for cancer treatment development. Cancer Chemother Pharmacol. 2015; 75(3):439-447.

24. Woller N, Gurlevik E, Ureche CI, Schumacher A, Kuhnel F. Oncolytic viruses as anticancer vaccines. Front Oncol. 2014;4:188.

25. Sieben M, Schafer P, Dinsart C, Galle PR, Moehler M. Activation of the human immune system via toll-like receptors by the oncolytic parvovirus H-1. Int J Cancer. 2013;132(11):2548-2556.

26. Prestwich RJ, Errington F, Ilett EJ, et al. Tumor infection by oncolytic reovirus primes adaptive antitumor immunity. Clin Cancer Res. 2008; 14(22):7358-7366.

27. Miyamoto $\mathrm{S}$, Inoue $\mathrm{H}$, Nakamura $\mathrm{T}$, et al. Coxsackievirus $\mathrm{B} 3$ is an oncolytic virus with immunostimulatory properties that is active against lung adenocarcinoma. Cancer Res. 2012;72(10):2609-2621.

28. Rommelfanger DM, Compte M, Diaz RM, et al. The efficacy versus toxicity profile of combination virotherapy and TLR immunotherapy highlights the danger of administering TLR agonists to oncolytic virustreated mice. Mol Ther. 2013;21(2):348-357.

29. Breitbach CJ, Arulanandam R, De Silva N, et al. Oncolytic vaccinia virus disrupts tumor-associated vasculature in humans. Cancer Res. 2013; 73(4):1265-1275.

30. Chiocca EA, Rabkin SD. Oncolytic viruses and their application to cancer immunotherapy. Cancer Immunol Res. 2014;2(4):295-300.

31. Taipale K, Liikanen I, Juhila J, et al. Chronic activation of innate immunity correlates with poor prognosis in cancer patients treated with oncolytic adenovirus. Mol Ther. 2016;(1):175-183.

32. Koks CA, Garg AD, Ehrhardt M, et al. Newcastle disease virotherapy induces long-term survival and tumor-specific immune memory in orthotopic glioma through the induction of immunogenic cell death. Int $J$ Cancer. 2015;136(5):E313-E325.

33. Cerullo V, Diaconu I, Romano V, et al. An oncolytic adenovirus enhanced for toll-like receptor 9 stimulation increases antitumor immune responses and tumor clearance. Mol Ther. 2012;20(11):2076-2086.

34. Grekova SP, Raykov Z, Zawatzky R, Rommelaere J, Koch U. Activation of a glioma-specific immune response by oncolytic parvovirus minute virus of mice infection. Cancer Gene Ther. 2012;19(7):468-475.

35. Wongthida P, Diaz RM, Pulido C, et al. Activating systemic T-cell immunity against self tumor antigens to support oncolytic virotherapy with vesicular stomatitis virus. Hum Gene Ther. 2011;22(11):1343-1353.

36. Zhang YQ, Tsai YC, Monie A, Wu TC, Hung CF. Enhancing the therapeutic effect against ovarian cancer through a combination of viral oncolysis and antigen-specific immunotherapy. Mol Ther. 2010;18(4): 692-699.

37. Bridle BW, Boudreau JE, Lichty BD, et al. Vesicular stomatitis virus as a novel cancer vaccine vector to prime antitumor immunity amenable to rapid boosting with adenovirus. Mol Ther. 2009;17(10):1814-1821.
38. Janelle V, Langlois MP, Lapierre P, Charpentier T, Poliquin L, Lamarre $\mathrm{A}$. The strength of the $\mathrm{T}$ cell response against a surrogate tumor antigen induced by oncolytic VSV therapy does not correlate with tumor control. Mol Ther. 2014;22(6):1198-1210.

39. Krysko DV, Garg AD, Kaczmarek A, Krysko O, Agostinis P, Vandenabeele P. Immunogenic cell death and DAMPs in cancer therapy. Nat Rev Cancer. 2012;12(12):860-875.

40. Kroemer G, Galluzzi L, Kepp O, Zitvogel L. Immunogenic cell death in cancer therapy. Annu Rev Immunol. 2013;31:51-72.

41. Aymeric L, Apetoh L, Ghiringhelli F, et al. Tumor cell death and ATP release prime dendritic cells and efficient anticancer immunity. Cancer Res. 2010;70(3):855-858.

42. Borde C, Barnay-Verdier S, Gaillard C, Hocini H, Marechal V, Gozlan J. Stepwise release of biologically active HMGB1 during HSV-2 infection. PLoS One. 2011;6(1):e16145.

43. Diaconu I, Cerullo V, Hirvinen ML, et al. Immune response is an important aspect of the antitumor effect produced by a CD40L-encoding oncolytic adenovirus. Cancer Res. 2012;72(9):2327-2338.

44. Donnelly OG, Errington-Mais F, Steele L, et al. Measles virus causes immunogenic cell death in human melanoma. Gene Ther. 2013; 20(1):7-15.

45. Bartlett DL, Liu Z, Sathaiah M, et al. Oncolytic viruses as therapeutic cancer vaccines. Mol Cancer. 2013;12(1):103.

46. Koks CA, De Vleeschouwer S, Graf N, Van Gool SW. Immune suppression during oncolytic virotherapy for high-grade glioma; yes or no? J Cancer. 2015;6(3):203-217.

47. Contag CH, Sikorski R, Negrin RS, et al. Definition of an enhanced immune cell therapy in mice that can target stem-like lymphoma cells. Cancer Res. 2010;70(23):9837-9845.

48. Gurlevik E, Woller N, Struver N, et al. Selectivity of oncolytic viral replication prevents antiviral immune response and toxicity, but does not improve antitumoral immunity. Mol Ther. 2010;18(11):1972-1982.

49. Parker KH, Sinha P, Horn LA, et al. HMGB1 enhances immune suppression by facilitating the differentiation and suppressive activity of myeloid-derived suppressor cells. Cancer Res. 2014;74(20): 5723-5733.

50. Liikanen I, Koski A, Merisalo-Soikkeli M, et al. Serum HMGB1 is a predictive and prognostic biomarker for oncolytic immunotherapy. Oncoimmunology. 2015;4(3):e989771.

51. Bollino D, Colunga A, Li B, Aurelian L. $\triangle \mathrm{PK}$ oncolytic activity includes modulation of tumor cell milieu. J Gen Virol. 2016;97(2):496-508.

52. Guo ZS, Liu Z, Bartlett DL. Oncolytic immunotherapy: dying the right way is a key to eliciting potent antitumor immunity. Front Oncol. 2014;4:74.

53. Senzer NN, Kaufman HL, Amatruda T, et al. Phase II clinical trial of a granulocyte-macrophage colony-stimulating factor-encoding, secondgeneration oncolytic herpesvirus in patients with unresectable metastatic melanoma. J Clin Oncol. 2009;27(34):5763-5771.

54. Kaufman HL, Kim DW, DeRaffele G, Mitcham J, Coffin RS, KimSchulze S. Local and distant immunity induced by intralesional vaccination with an oncolytic herpes virus encoding GM-CSF in patients with stage IIIc and IV melanoma. Ann Surg Oncol. 2010;17(3): 718-730.

55. Heo J, Reid T, Ruo L, et al. Randomized dose-finding clinical trial of oncolytic immunotherapeutic vaccinia JX-594 in liver cancer. Nat Med. 2013;19(3):329-336.

56. Kim MK, Breitbach CJ, Moon A, et al. Oncolytic and immunotherapeutic vaccinia induces antibody-mediated complement-dependent cancer cell lysis in humans. Sci Transl Med. 2013;5(185):185ra163.

57. Cerullo V, Pesonen S, Diaconu I, et al. Oncolytic adenovirus coding for granulocyte macrophage colony-stimulating factor induces antitumoral immunity in cancer patients. Cancer Res. 2010;70(11):4297-4309.

58. Koski A, Kangasniemi L, Escutenaire S, et al. Treatment of cancer patients with a serotype $5 / 3$ chimeric oncolytic adenovirus expressing GMCSF. Mol Ther. 2010;18(10):1874-1884.

59. Atherton MJ, Lichty BD. Evolution of oncolytic viruses: novel strategies for cancer treatment. Immunotherapy. 2013;5(11):1191-1206. 
60. Gil M, Seshadri M, Komorowski MP, Abrams SI, Kozbor D. Targeting CXCL12/CXCR4 signaling with oncolytic virotherapy disrupts tumor vasculature and inhibits breast cancer metastases. Proc Natl Acad Sci U S A. 2013;110(14):E1291-E1300.

61. Gil M, Komorowski MP, Seshadri M, et al. CXCL12/CXCR4 blockade by oncolytic virotherapy inhibits ovarian cancer growth by decreasing immunosuppression and targeting cancer-initiating cells. J Immunol. 2014;193(10):5327-5337.

62. Bhattacharya P, Budnick I, Singh M, et al. Dual role of GM-CSF as a pro-inflammatory and a regulatory cytokine: implications for immune therapy. J Interferon Cytokine Res. 2015;35(8):585-599.

63. Galivo F, Diaz RM, Thanarajasingam U, et al. Interference of CD40Lmediated tumor immunotherapy by oncolytic vesicular stomatitis virus. Hum Gene Ther. 2010;21(4):439-450.

64. Devaud C, John LB, Westwood JA, Darcy PK, Kershaw MH. Immune modulation of the tumor microenvironment for enhancing cancer immunotherapy. Oncoimmunology. 2013;2(8):e25961.

65. Whiteside TL. Tumor-induced death of immune cells: its mechanisms and consequences. Semin Cancer Biol. 2002;12(1):43-50.

66. Serrano AE, Menares-Castillo E, Garrido-Tapia M, et al. Interleukin 10 decreases MICA expression on melanoma cell surface. Immunol Cell Biol. 2011;89(3):447-457.

67. Ben-Shoshan J, Maysel-Auslender S, Mor A, Keren G, George J. Hypoxia controls CD4+CD25+regulatory T-cell homeostasis via hypoxia-inducible factor-1alpha. Eur J Immunol. 2008;38(9):2412-2418.

68. Noman MZ, Desantis G, Janji B, et al. PD-L1 is a novel direct target of HIF-1alpha, and its blockade under hypoxia enhanced MDSC-mediated T cell activation. $J$ Exp Med. 2014;211(5):781-790.

69. Marvel D, Gabrilovich DI. Myeloid-derived suppressor cells in the tumor microenvironment: expect the unexpected. J Clin Invest. 2015; 125(9):3356-3364.

70. Zou W. Immunosuppressive networks in the tumour environment and their therapeutic relevance. Nat Rev Cancer. 2005;5(4):263-274.

71. Roy DG, Bell JC. Cell carriers for oncolytic viruses: current challenges and future directions. Oncolytic Virother. 2013;2:47-56.

72. Tyler MA, Ulasov IV, Sonabend AM, et al. Neural stem cells target intracranial glioma to deliver an oncolytic adenovirus in vivo. Gene Ther. 2009;16(2):262-278.

73. Kirn DH, Thorne SH. Targeted and armed oncolytic poxviruses: a novel multi-mechanistic therapeutic class for cancer. Nat Rev Cancer. 2009;9:64-71.

74. Jennings VA, Ilett EJ, Scott KJ, et al. Lymphokine-activated killer and dendritic cell carriage enhances oncolytic reovirus therapy for ovarian cancer by overcoming antibody neutralization in ascites. Int J Cancer. 2014;134:1091-1101.

75. Iankov I, Msaouel P, Allen C, et al. Demonstration of anti-tumor activity of oncolytic measles virus strains in a malignant pleural effusion breast cancer model. Breast Cancer Res Treat. 2010;122:745-754.

76. Ilett EJ, Prestwich RJ, Kottke T, et al. Dendritic cells and T cells deliver oncolytic reovirus for tumour killing despite pre-existing anti-viral immunity. Gene Ther. 2009;16:689-699.
77. Leveille S, Goulet ML, Lichty BD, Hiscott J. Vesicular stomatitis virus oncolytic treatment interferes with tumor-associated dendritic cell functions and abrogates tumor antigen presentation. $J$ Virol. 2011;85: $12160-12169$.

78. Ong HT, Federspiel MJ, Guo CM, et al. Systemically delvirus-infected mesenchymal stem cells can evade host immunity to inhibit liver cancer growth. J Hepatol. 2013;59(5):999-1006.

79. Bridle BW, Chen L, Lemay CG, et al. HDAC inhibition suppresses primary immune responses, enhances secondary immune responses, and abrogates autoimmunity during tumor immunotherapy. Mol Ther. 2013;21(4):887-894.

80. Alvarez-Breckenridge CA, Yu J, Price R, et al. NK cells impede glioblastoma virotherapy through NKp30 and NKp46 natural cytotoxicity receptors. Nat Med. 2012;18(12):1827-1834.

81. Khan AN, Gregorie CJ, Tomasi TB. Histone deacetylase inhibitors induce TAP, LMP, Tapasin genes and MHC class I antigen presentation by melanoma cells. Cancer Immunol Immunother. 2008;57(5): 647-654.

82. Cerullo V, Diaconu I, Kangasniemi L, et al. Immunological effects of low-dose cyclophosphamide in cancer patients treated with oncolytic adenovirus. Mol Ther. 2011;19(9):1737-1746.

83. Doloff JC, Waxman DJ. VEGF receptor inhibitors block the ability of metronomically dosed cyclophosphamide to activate innate immunityinduced tumor regression. Cancer Res. 2012;72(5):1103-1115.

84. Kottke T, Galivo F, Wongthida P, et al. Treg depletion-enhanced IL-2 treatment facilitates therapy of established tumors using systemically delivered oncolytic virus. Mol Ther. 2008;16(7):1217-1226.

85. Ghiringhelli F, Menard C, Puig PE, et al. Metronomic cyclophosphamide regimen selectively depletes $\mathrm{CD} 4+\mathrm{CD} 25+$ regulatory $\mathrm{T}$ cells and restores $\mathrm{T}$ and $\mathrm{NK}$ effector functions in end stage cancer patients. Cancer Immunol Immunother. 2007;56(5):641-648.

86. Esaki S, Goshima F, Kimura H, Murakami S, Nishiyama Y. Enhanced antitumoral activity of oncolytic herpes simplex virus with gemcitabine using colorectal tumor models. Int J Cancer. 2013;132(7):1592-1601.

87. Nguyen A, Ho L, Wan Y. Chemotherapy and oncolytic virotherapy: advanced tactics in the war against cancer. Front Oncol. 2014;4:145.

88. John LB, Howland LJ, Flynn JK, et al. Oncolytic virus and anti-4$1 \mathrm{BB}$ combination therapy elicits strong antitumor immunity against established cancer. Cancer Res. 2012;72(7):1651-1660.

89. Coffin RS. From virotherapy to oncolytic immunotherapy: where are we now? Curr Opin Virol. 2015;13:93-100.

90. Workenhe ST, Simmons G, Pol JG, Lichty BD, Halford WP, Mossman KL. Immunogenic HSV-mediated oncolysis shapes the antitumor immune response and contributes to therapeutic efficacy. Mol Ther. 2014;22(1):123-131.

91. Devarapu SK, Mamidi S, Plöger F, Dill O, Blixt O, Kirschfink M, Schwartz-Albiez R. Cytotoxic Activity Against Human Neuroblastoma and Melanoma Cells Mediated by IgM Antibodies Derived from Peripheral Blood of Healthy Donors. Int J Cancer. Epub 2016 Feb 23.
OncoTargets and Therapy

\section{Publish your work in this journal}

OncoTargets and Therapy is an international, peer-reviewed, open access journal focusing on the pathological basis of all cancers, potential targets for therapy and treatment protocols employed to improve the management of cancer patients. The journal also focuses on the impact of management programs and new therapeutic agents and protocols on

\section{Dovepress}

patient perspectives such as quality of life, adherence and satisfaction. The manuscript management system is completely online and includes a very quick and fair peer-review system, which is all easy to use. Visit http://www.dovepress.com/testimonials.php to read real quotes from published authors. 